\title{
Plexiform Fibrohistiocytic Tumor on the Ear: Case Report and Immunohistochemical Investigation of Stromal Factor
}

\author{
Kosuke Shido Taku Fujimura Aya Kakizaki Sadanori Furudate \\ Masayuki Asano Setsuya Aiba \\ Department of Dermatology, Tohoku University Graduate School of Medicine, Sendai, \\ Japan
}

\section{Key Words}

Plexiform fibrohistiocytic tumor - Periostin - Tumor-associated macrophages .

Immunomodulation

\begin{abstract}
Plexiform fibrohistiocytic tumor (PFT) is a rare mesenchymal neoplasm of intermediate malignant potential with a high local recurrence rate. In this report, we describe a case of PFT on the ear, which showed a dense deposition of periostin (POSTN) in the stromal areas of the tumor. In addition, dense infiltration of CD163+CD206- tumor-associated macrophages (TAMs) was detected in the same areas as POSTN. Since POSTN was previously reported to possess immunomodulatory effects on TAMs, our present report suggested the significance of the POSTN/TAMs axis in the progression of PFT.

(C) 2016 The Author(s)

Published by S. Karger AG, Basel
\end{abstract}

\section{Introduction}

Plexiform fibrohistiocytic tumor (PFT) presents as a slowly developing nodule on the extremities and trunk [1, 2], and generally occurs in children and young adults [1, 2]. Although approximately 150 cases have been presented in English language papers about PFT [1-4], little is known about its pathogenesis.

\section{KARGER}

Taku Fujimura, MD

Department of Dermatology

Tohoku University Graduate School of Medicine

Seiryo-machi 1-1, Aoba-ku, Sendai 980-8574 (Japan)

E-Mail tfujimura1@mac.com 
Case Reports in
Dermatology

\begin{tabular}{l|l}
\hline Case Rep Dermatol 2016;8:26-30 \\
\hline DOI: 10.1159/000444045 & $\begin{array}{l}\text { ○ 2016 The Author(s). Published by S. Karger AG, Basel } \\
\text { www.karger.com/cde }\end{array}$ \\
\hline
\end{tabular}

Shido et al.: Plexiform Fibrohistiocytic Tumor on the Ear: Case Report and Immunohistochemical Investigation of Stromal Factor

\section{Case Report}

A 28-year-old Japanese woman visited our outpatient clinic with a 1.5-year history of an asymptomatic nodule developing on her left retroauricular skin. Her tumor had been resected by a general surgeon as a benign spindle cell tumor without further histological diagnosis 1 year before. On her initial visit, physical examination revealed a well-demarcated nodule on her retroauricular skin, $20 \times 20 \mathrm{~mm}$ in size (fig. 1a). Magnetic resonance imaging revealed a subcutaneous nodule located adjacent to the periosteum (fig. 1b). We excised the nodule together with the adjacent periosteum with a 5-mm surgical margin. The histopathological findings revealed proliferation of fibroblastic and histiocytic cells showing storiform-type whorling and a plexiform pattern (fig. 1c, d). These tumor-composing spindle cells were positive for CD68 and SMA, and negative for S100 and factor XIIIa. The Ki67 score was approximately $10 \%$. Based on the above finding, we diagnosed this tumor as a PFT. The differential diagnoses of PFT are listed in table 1 [2].

Since the pathogenesis and biological behavior of PFT are still incompletely understood, we employed immunohistochemical staining for periostin (POSTN), CD163 and CD206 to further investigate the possible immunological background of the PFT. A dense deposition of POSTN was detected throughout the stromal areas of the tumor (fig. 2a). In addition, CD163+CD206- tumor-associated macrophages (TAMs) were densely infiltrated in the same areas of POSTN (fig. 2b, c).

\section{Discussion}

In this report, we describe a case of PFT on the ear which showed a dense deposition of POSTN in the stromal areas of the tumor. PFT is a rare mesenchymal neoplasm of intermediate malignant potential with a high local recurrence rate, like dermatofibrosarcoma protuberans [1-4]. Immunohistochemical staining is helpful for the differential diagnosis of PFT $[1,2]$ : e.g. it is positive for CD68, vimentin and NSE, and negative for S100, CD34, factor XIIIa and calponin $[2,3]$. Since these diagnostic markers for PFT do not reflect the biological behavior of this type of malignant tumor, in this report we employed immunohistochemical staining for POSTN as well as CD163 and CD206, focusing on the cancer stroma of PFT to investigate the possible mechanisms of local invasion and recurrence of PFT.

POSTN is an extracellular matrix protein that is involved in modulating cell functions through various pathways [5-7]. In the dermatological field, the deposition of POSTN is reported in various skin diseases, including fibrosing diseases such as keloid, hypertrophic scar [8], morphea and lichen sclerosis et atrophicus [9]. Zhou et al. [6] reported the importance of POSTN in developing glioblastoma multiforme by the induction of M2-like TAMs in the tumor microenvironment. In addition, as we previously reported, POSTN stimulates CD163+CD206- monocyte-derived macrophages to produce a series of chemokines that might be connected with the cancer formation of mycosis fungoides [7]. Notably, this study also suggests that CD163+CD206- monocyte-derived macrophages augment the mRNA expression of MMP12 by POSTN stimulation. Interestingly, MMP12 is reported to be increased in the serum and skin lesions of systemic sclerosis, and is associated with the severity of skin and pulmonary fibrosis [10]. These reports suggested the significance of the POSTN/TAMs axis in the progression of various types of tumors, and suggested that POSTN stimulation might modulate the function of CD163+CD206- TAMs to develop the fibrosis in PFT. To confirm this hypothesis, further cases and studies will be necessary. 
Shido et al.: Plexiform Fibrohistiocytic Tumor on the Ear: Case Report and Immunohistochemical Investigation of Stromal Factor

\section{Statement of Ethics}

The patient gave written informed consent.

\section{Disclosure Statement}

The authors have no conflicting interests to declare.

\section{References}

1 Lynnhtun K, Achan A, Shingde M, Chou S, Howle JR, Sharma R: Plexiform fibrohistiocytic tumour: morphological changes and challenges in assessment of recurrent and metastatic lesions. Histopathology 2012;60:1156-1158.

72 Taher A, Pushpanathan C: Plexiform fibrohistiocytic tumor: a brief review. Arch Pathol Lab Med 2007;131: 1135-1138.

3 Jaffer S, Ambrosini-Spaltro A, Mancini AM, Eusebi V, Rosai J: Neurothekeoma and plexiform fibrohistiocytic tumor: mere histologic resemblance or histogenetic relationship? Am J Surg Pathol 2009;33:905-913.

-4 Muezzinoglu B, Tohumcu A, Ekingen G: An unusual occurrence of plexiform fibrohistiocytic tumour: congenital tumour diagnosed at 7 years of age. Pathology 2011;43:380-381.

-5 Ando T, Xiao W, Gao P, Namiranian S, Matsumoto K, Tomimori Y, Hong H, Yamashita H, Kimura M, Kashiwakura J, Hata TR, Izuhara K, Gurish MF, Roers A, Rafaels NM, Barnes KC, Jamora C, Kawakami Y, Kawakami T: Critical role for mast cell Stat5 activity in skin inflammation. Cell Rep 2014;6:366-376.

6 Zhou W, Ke SQ, Huang Z, Flavahan W, Fang X, Paul J, Wu L, Sloan AE, McLendon RE, Li X, Rich JN, Bao S: Periostin secreted by glioblastoma stem cells recruits M2 tumour-associated macrophages and promotes malignant growth. Nat Cell Biol 2015;17:170-182.

-7 Furudate S, Fujimura T, Kakizaki A, Kambayashi Y, Asano M, Watabe A, Aiba S: The possible interaction between periostin expressed by cancer stroma and tumor-associated macrophages in developing mycosis fungoides. Exp Dermatol 2016;25:107-112.

8 Sidgwick GP, Bayat A: Extracellular matrix molecules implicated in hypertrophic and keloid scarring. J Eur Acad Dermatol Venereol 2012;26:141-152.

$\checkmark 9$ Kakizaki A, Fujimura T, Furudate S, Kambayashi Y, Aiba S: Lichen sclerosus et atrophicus on the abdomen accompanied by localized scleroderma. Case Rep Dermatol 2015;7:39-45.

10 Manetti M, Guiducci S, Romano E, Bellando-Randone S, Conforti ML, Ibba-Manneschi L, Matucci-Cerinic M Increased serum levels and tissue expression of matrix metalloproteinase-12 in patients with systemic sclerosis: correlation with severity of skin and pulmonary fibrosis and vascular damage. Ann Rheum Dis 2012;71:1064-1072.

Table 1. Differential diagnoses of PFT

How to differentiate from PFT

Plexiform neurofibroma S100+

Plexiform schwannoma S100+

Cellular neurothekeoma S100+

Fibrous hamartoma of infancy possesses the primitive cellular myxoid component

Benign fibrous histiocytoma solid-pattern tumor

Dermatofibroma storiform-pattern tumor

Myofibromatosis well-demarcated with a zoned appearance 


\section{Case Reports in Dermatology}

Shido et al.: Plexiform Fibrohistiocytic Tumor on the Ear: Case Report and Immunohistochemical Investigation of Stromal Factor
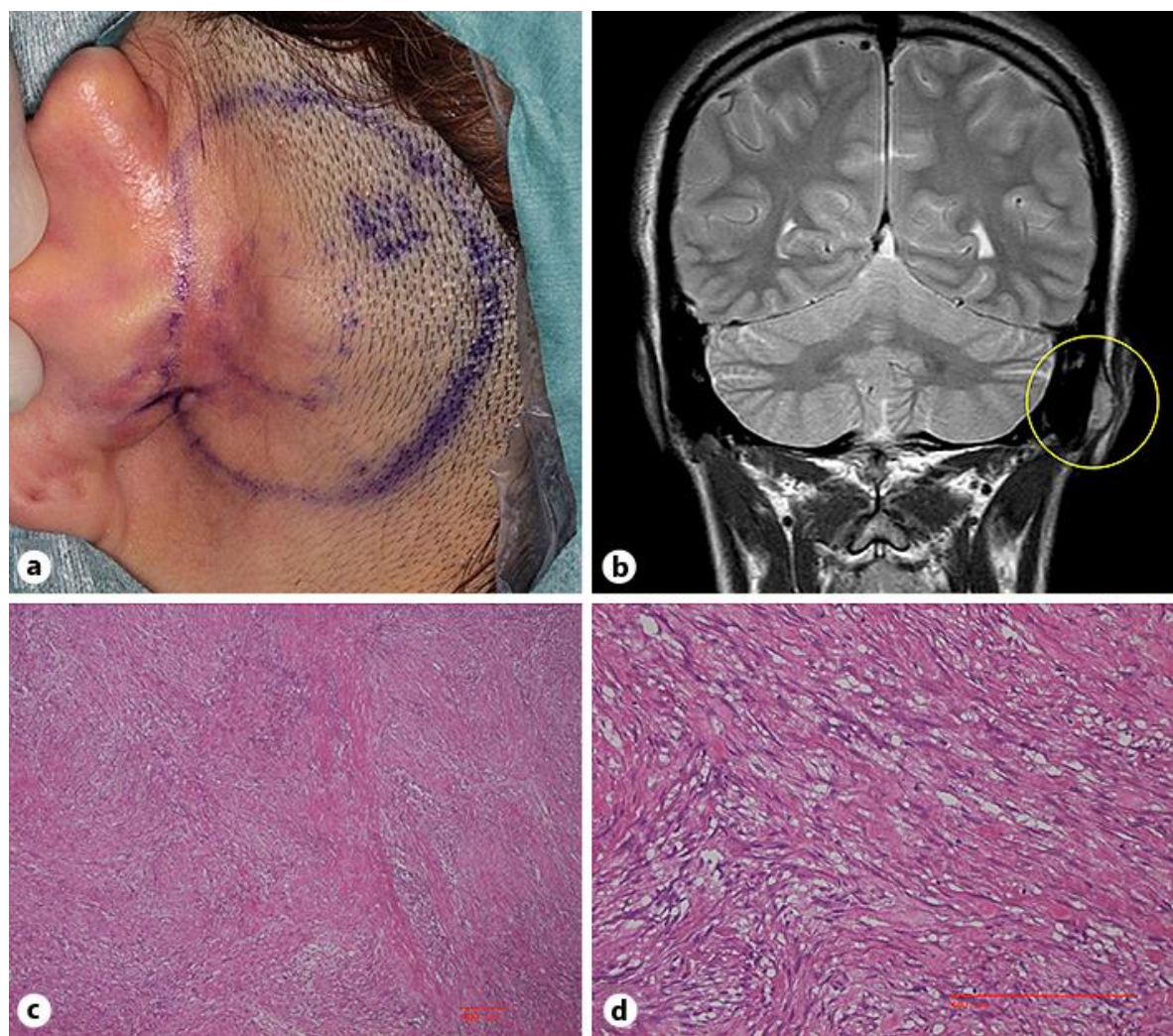

Fig. 1. a A well-demarcated nodule on the retroauricular skin, $20 \times 20 \mathrm{~mm}$ in size. b Magnetic resonance imaging revealed a subcutaneous nodule located adjacent to the periosteum. c, d Proliferation of fibroblastic and histiocytic cells showing storiform-type whorling and a plexiform pattern. Original magnification: $\times 100(c), \times 400(d)$. 
Shido et al.: Plexiform Fibrohistiocytic Tumor on the Ear: Case Report and Immunohistochemical Investigation of Stromal Factor
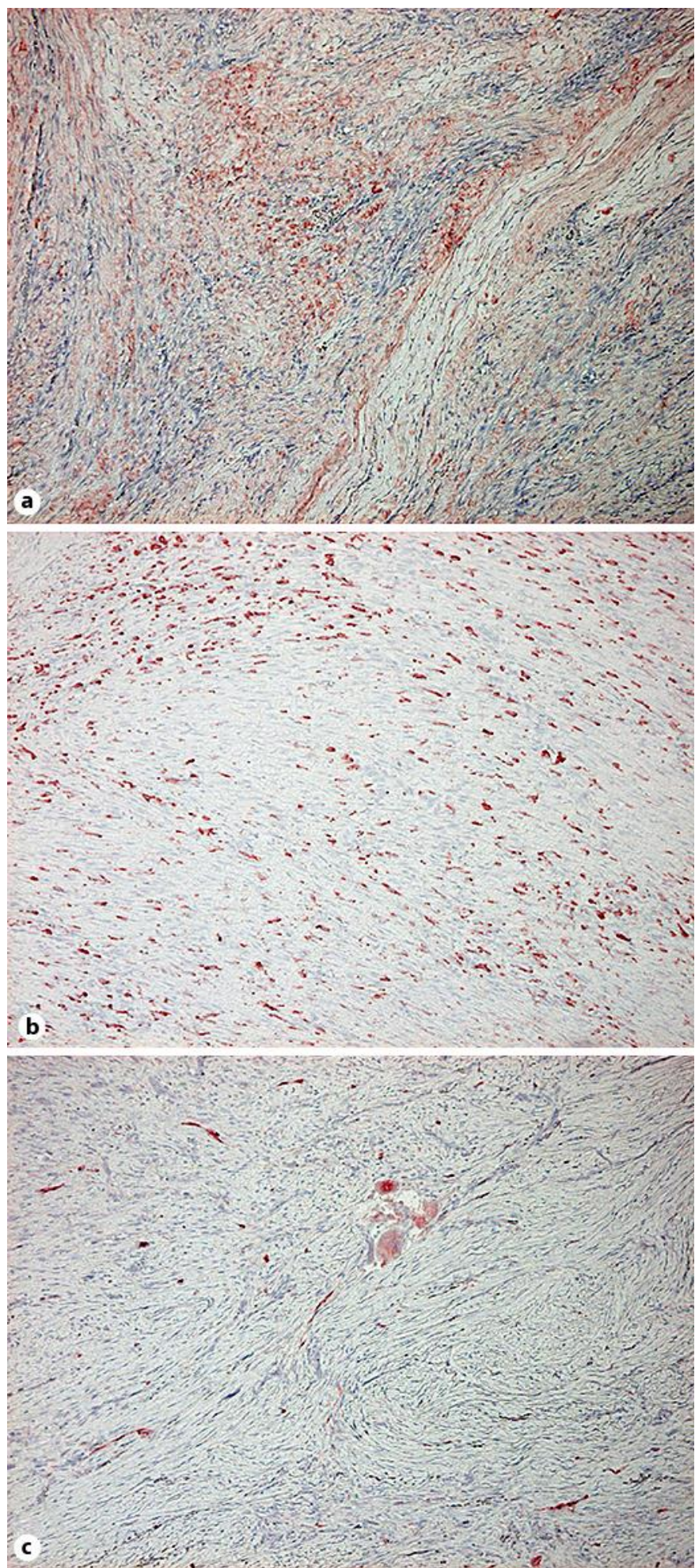

Fig. 2. Paraffin-embedded tissue samples were deparaffinized and stained with anti-POSTN antibody (a), anti-CD163 antibody (b) and anti-CD206 antibody (c). The sections were developed with liquid permanent red. Original magnification: $\times 200$. 